\title{
Comparative analysis of involvement of UGT1 and UGT2 splice variants of UDP-galactose transporter in glycosylation of macromolecules in MDCK and CHO cell lines
}

\author{
Dorota Maszczak-Seneczko • Teresa Olczak • \\ Livius Wunderlich • Mariusz Olczak
}

Received: 21 June 2011 /Revised: 18 August 2011 / Accepted: 19 August 2011 /Published online: 6 September 2011

(C) The Author(s) 2011. This article is published with open access at Springerlink.com

\begin{abstract}
Nucleotide sugar transporters deliver nucleotide sugars into the Golgi apparatus and endoplasmic reticulum. This study aimed to further characterize mammalian UDP-galactose transporter (UGT) in MDCK and $\mathrm{CHO}$ cell lines. MDCK-RCA ${ }^{\mathrm{r}}$ and CHO-Lec8 mutant cell lines are defective in UGT transporter, although they exhibit some level of galactosylation. Previously, only single forms of UGT were identified in both cell lines, UGT1 in MDCK cells and UGT2 in CHO cells. We have identified the second UGT splice variants in CHO (UGT1) and MDCK (UGT2) cells. Compared to UGT1, UGT2 is more abundant in nearly all examined mammalian tissues and cell lines, but MDCK cells exhibit different relative distribution of both splice variants. Complementation analysis demonstrated that both UGT splice variants are necessary for $\mathrm{N}$ - and $\mathrm{O}$-glycosylation of proteins. Both mutant cell lines produce chondroitin-4sulfate at only a slightly lower level compared to wild-type cells. This defect is corrected by overexpression of both UGT splice variants. MDCK-RCA ${ }^{\mathrm{r}}$ mutant cells do not produce keratan sulfate and this effect is not corrected by
\end{abstract}

Electronic supplementary material The online version of this article (doi:10.1007/s10719-011-9348-z) contains supplementary material, which is available to authorized users.

D. Maszczak-Seneczko $\cdot$ T. Olczak $\cdot$ M. Olczak $(\bowtie)$

Laboratory of Biochemistry, Faculty of Biotechnology,

University of Wroclaw,

Tamka 2, 50-137 Wroclaw, Poland

e-mail: Mariusz.Olczak@biotech.uni.wroc.pl

L. Wunderlich

Laboratory of Biochemistry and Molecular Biology,

Department of Applied Biotechnology and Food Science,

Budapest University of Technology and Economics,

1521 Budapest, P.O. Box 91, Hungary either UGT splice variant, overexpressed either singly or in combination. Here we demonstrate that both UGT splice variants are important for glycosylation of proteins. In contrast to MDCK cells, MDCK-RCA ${ }^{\mathrm{r}}$ mutant cells may possess an additional defect within the keratan sulfate biosynthesis pathway.

Keywords CHO-Lec8 - Glycosylation · MDCK-RCA ${ }^{\mathrm{r}}$. Splice variant $\cdot$ UDP-galactose transporter
Abbreviations
ER Endoplasmic reticulum
NST Nucleotide sugar transporter
UGT UDP-galactose transporter
UGT1 UDP-galactose transporter splice variant 1 localized to the Golgi apparatus
UGT2 UDP-galactose transporter splice variant 2 localized to the Golgi apparatus and ER

\section{Introduction}

Cellular glycoconjugates play a variety of fundamental roles in the growth and development of eukaryotes, as well as in the cell surface recognition of hosts by pathogens. The glycan moiety is synthesized and modified by glycosyltransferases located in the lumen of the endoplasmic reticulum (ER) and Golgi apparatus. The substrates required by glycosyltransferases are sugars activated by the addition of a nucleoside mono- or diphosphate (UDP, GDP, or CMP). Nucleotide sugars are synthesized in the cytosol [1], except for CMP-sialic acid, which is synthesized in the nucleus [2]. To be available for glycosyltransferases, they must be transported into the 
ER or Golgi apparatus. This function is played by nucleotide sugar transporters (NSTs) [for reviews see 3-5], which are hydrophobic proteins with a molecular weight of 30-45 kDa. Most predictions determine in these multitransmembrane-spanning proteins an even number of spans, which results in the $\mathrm{N}$ and $\mathrm{C}$ termini being directed to the cytosolic side of the membrane. Membrane topology has been determined for the murine CMP-sialic acid transporter only and, in contrast to the theoretically predicted eight transmembrane domains, ten transmembrane domains have been experimentally identified [6]. NSTs function in the form of dimers [6-10] or higher oligomers [11]. It has been demonstrated that they act as antiporters, exchanging the nucleotide sugar with the corresponding nucleoside monophosphate, which is a product of the glycosylation reaction [3-5].

The first characterized NSTs were specific for the translocation of a single nucleotide sugar [for review see 4, 5]. Recently, multisubstrate transporters of nucleotide sugars have been described in several organisms, including Leishmania donovani [11], Drosophila melanogaster [12-14], Entamoeba histolytica [15], Caenorhabditis elegans [16-18], and humans [19-21].

UDP-galactose transporting activity has been reported in several organisms [16, 22-29]. Detailed characterization of UDP-galactose transporter (UGT) was possible after mutant cell lines, such as MDCK-RCA ${ }^{\mathrm{r}}[28,29]$, CHO Lec-8 [25, 30-32], and Had-1 [33], had been generated. Nonsense mutations identified in the mutant cells cause inhibition of UGT production, resulting in macromolecules deficient in galactose [28, 30-38]. Complementation of this defect results in reconstituted UDP-galactose transport. Expression of recombinant UGT in mammalian and yeast cells also confirmed its localization and specificity [22, 24, 26, 27].

Previously, two UGT (SLC35A2) isoforms (UGT1 and UGT2) have been identified in humans [22, 24], but only single orthologs have been found in the CHO cell line [25], MDCK cell line [34], and in mouse [35]. The mouse and MDCK UGTs resembled human UGT1, while the CHO gene encoded a protein most homologous to human UGT2. The human UGT gene contains five exons, with stop codons in exons 4 and 5 [25]. Translation of exons 1 to 4 results in UGT1. If a splice consensus motif present in exon 4 is used, exon 5 becomes fused to the $5^{\prime}$ region of exon 4 , and UGT2 is translated. Although NSTs have been mainly identified in the Golgi apparatus, those located in the ER have also been characterized, including UDP$\mathrm{N}$-acetylglucosamine transporter of Saccharomyces cerevisiae [39-41] or UGT of Schizosaccharomyces pombe and S. cerevisiae [41]. It has been shown that UGT1-like isoform localizes to the Golgi apparatus, whereas UGT2-like isoform localizes to both the Golgi apparatus and ER [42]. Analysis of both UGT splice variants has demonstrated that differences in human UGT isoforms are confined to the C terminus. The dilysine motif present at the $\mathrm{C}$ terminus of UGT2 has been shown to be responsible for its dual localization $[25,42]$. In the MDCK cell line, in both wildtype and mutant cells, as well as in canine leukocytes, an additional shorter UGT isoform has also been identified and characterized [34]. It contains an in-frame 183-bp deletion close to the $\mathrm{N}$ terminus of the UGT. In contrast, this splice variant has not been found in human cell lines (i.e. HL-60, HeLa), human tissues or the $\mathrm{CHO}$ cell line [34].

In an attempt to further characterize the UDPgalactose transporter, we identified the second UGT splice variant in CHO (UGT1-like isoform) and MDCK (UGT2-like isoform) cell lines and examined glycosylation profiles of the wild-type and mutant MDCK-RCA ${ }^{\mathrm{r}}$ and CHO-Lec8 cells before and after phenotypic correction by overexpression of the respective human UGT isoforms. This allowed comparison of both UGT splice variants' involvement in glycosylation of macromolecules.

\section{Materials and methods}

Rapid amplification of cDNA ends (RACE)

NucleoSpin RNA II Kit (Macherey-Nagel) was used to purify total RNA from MDCK and CHO cells. The concentration of purified RNA was determined spectrophotometrically at $260 \mathrm{~nm}$. The quality and quantity of purified RNA were further examined using an Agilent 2100 Bioanalyzer equipped with RNA Chip (Agilent Technologies) (Supplementary Fig. S1). RACE was performed using the GeneRacer Kit (Invitrogen). One nested PCR with MDCK cDNA and two nested PCRs with CHO cDNA were performed to isolate specific products encoding UGT splice variants. Analysis of sequences coding for other proteins was carried out using the same procedure. Primers used in this experiment are listed in Table 1. OptiTaq polymerase (Eurx) was used in all reactions. RACE fragments were purified, ligated to pJet1.2 Blunt Vector (CloneJet PCR Cloning Kit, Fermentas) and sequenced (Genomed).

Reverse transcription-polymerase chain reaction (RT-PCR)

For RT-PCR reactions, Titan One Tube RT-PCR System (Roche) was used. As templates, total RNA samples obtained from 20 human tissues (Ambion), total RNA purified from 9 human cell lines, Madin-Darby canine kidney II (MDCK) and Chinese hamster ovary (CHO) cell lines were used. The concentration of purified RNA was determined spectrophotometrically at $260 \mathrm{~nm}$. The quality and quantity of purified RNA were further examined using 
Table 1 Primers used for 3'RACE reactions and RT-PCR

\begin{tabular}{lll}
\hline Primer & Sequence $\left(5^{\prime} \rightarrow 3^{\prime}\right)$ & Description \\
\hline 3RacerCHOGal1 & GGTGGGCTACTGGTGGCTGTTGTAGT & 3'RACE CHO 1st primer \\
3RacerCHOGal2 & CCTGGGCGCTGGGCTCGTCATTGG & 3'RACE CHO 2nd primer \\
3RacerCHOGal3 & CCTCTGCCTCTGGGCCCTGCATTCA & 3'RACE CHO 3rd primer \\
3RacerMDCKGal1 & GCTTCCACGTGGACCCATTGTTTGC & 3'RACE MDCK 1st primer \\
3RacerMDCKGal2 & GCCAAAGCCATAGCCTCCACCTCC & 3'RACE MDCK 2nd primer \\
HsGalUni-F & CTACTGGTGGCTGTGGTTGTC & RT-PCR of human UGT splice variants \\
HsGalER-R & GAACCCTTCACCTTGGTGAGC & RT-PCR of human UGT2 splice variant \\
HsGalGolgi-R & CCTCACTTCACCAGCACTGAC & RT-PCR of human UGT1 splice variant \\
CfGalUni-F & GGCTGCTGGTGGCTGTTGTCGT & RT-PCR of MDCK UGT splice variants $\left(\mathrm{T}_{\mathrm{m}}=60.4^{\circ} \mathrm{C}\right)$ \\
CfGalER-R & CTACGAACCCTTCACCTTGGTGAGC & RT-PCR of MDCK UGT2 splice variant $\left(\mathrm{T}_{\mathrm{m}}=61^{\circ} \mathrm{C}\right)$ \\
CfGalGolgi-R & TCACTTCACCAGCACTGACTTCGG & RT-PCR of MDCK UGT1 splice variant $\left(\mathrm{T}_{\mathrm{m}}=59.1^{\circ} \mathrm{C}\right)$ \\
CgGalUni-F & GGCTACTGGTGGCTGTTGTAGTC & RT-PCR of CHO UGT splice variants $\left(\mathrm{T}_{\mathrm{m}}=58.8^{\circ} \mathrm{C}\right)$ \\
CgGalER-R & CGAACCCTTCACCTTGGTGAGCA & RT-PCR of CHO UGT2 splice variant $\left(\mathrm{T}_{\mathrm{m}}=58.8^{\circ} \mathrm{C}\right)$ \\
CgGalGolgi-R & GCTCTCACTTCACCAGCACTGAC & RT-PCR of CHO UGT1 splice variant $\left(\mathrm{T}_{\mathrm{m}}=58.8^{\circ} \mathrm{C}\right)$ \\
GAPDH-F & TGCACCACCAACTGCTTAGC & RT-PCR of glyceraldehyde-3-phosphate dehydrogenase $(\mathrm{GAPDH})[43]$ \\
GAPDH-R & GGCATGGACTGTGGTCATGAG & RT-PCR of glyceraldehyde-3-phosphate dehydrogenase $(\mathrm{GAPDH})[43]$ \\
B2M-F & TGCTGTCTCCATGTTTGATGTATCT & RT-PCR of beta-2-microglobulin $(\mathrm{B} 2 \mathrm{M})[43]$ \\
B2M-R & TCTCTGCTCCCCACCTCTAAGT & RT-PCR of beta-2-microglobulin $(\mathrm{B} 2 \mathrm{M})[43]$ \\
\hline
\end{tabular}

an Agilent 2100 Bioanalyzer equipped with RNA Chip (Supplementary Fig. S1). All RNA samples isolated from cell lines were digested with DNase. RNA samples derived from human tissues, purchased from Ambion, were certified as DNA free. Expression levels of beta-2microglobulin (B2M) as a housekeeping gene were determined [43]. Because two human cell lines did not produce its transcript, expression levels of glyceraldehyde-3phosphate dehydrogenase (GAPDH) were determined [43]. Primers used in this experiment are listed in Table 1. Primers used to amplify UGT splice variants in $\mathrm{CHO}$ and MDCK cells were designed to have identical or almost identical $\mathrm{T}_{\mathrm{m}}$ and $\mathrm{G}+\mathrm{C}$ contents (Table 1), which resulted in specific, distinct products at desired lengths after RT-PCR. After initial denaturation $\left(65^{\circ} \mathrm{C}, 5 \mathrm{~min}\right)$, RT was carried out using $1 \mu \mathrm{g}$ of total RNA at $55^{\circ} \mathrm{C}$ for $30 \mathrm{~min}$. Amplified DNA fragments specific for UGT1 and UGT2 were synthesized for 30 cycles of PCR at $94^{\circ} \mathrm{C}$ for $15 \mathrm{~s}$ (denaturation), $55^{\circ} \mathrm{C}$ for $30 \mathrm{~s}$ (annealing), and $68^{\circ} \mathrm{C}$ for 1 min (elongation), separated in $1.4 \%$ agarose gel and visualized with ethidium bromide.

Construction of mammalian expression plasmids

Open reading frames (ORFs) of human UGT1 and UGT2 (both containing stop codons) with appropriate restriction sites at both ends were amplified using cDNA synthesized from $5 \mu \mathrm{g}$ of total RNA as a template. RNA was isolated from $2 \times 10^{6}$ HL-60 cells using the NucleoSpin RNA II Kit. For the RT reaction, the Thermoscript 1st Strand Synthesis Kit (Invitrogen) and oligo $\mathrm{dT}(20)$ were used in conditions recommended by the manufacturer. Constructs containing the FLAG epitope at the $\mathrm{N}$ terminus were prepared by ligation of UGT1 and UGT2 encoding sequences into 3xFLAG-myc-CMV-26 (Sigma). For pVitro1-neo plasmid (InvivoGen), first adaptors encoding 6His-c-myc epitopes (MCS1) and 6His-HA epitopes (MCS2) were ligated. All adaptors were prepared from sense and antisense oligonucleotides (Genomed) purified using denaturing polyacrylamide gel electrophoresis and annealed at an initial temperature of $80^{\circ} \mathrm{C}$ with slow cooling down $\left(1^{\circ} \mathrm{C}\right.$ per $\min )$ to $35^{\circ} \mathrm{C}$ in $0.5 \mathrm{M} \mathrm{NaCl}$. Sequences of adaptor oligonucleotides and primers for amplification of ligated ORFs are available upon request. Constructs containing short epitopes at the start of each polylinker were used for ligation of UGT1 and UGT2 encoding sequences. All ligations were performed using the Rapid DNA Ligation Kit (Fermentas). Additional sequences attached to the $\mathrm{N}$ terminus of UGT1 and UGT2 proteins contain the respective epitopes and amino acid residues resulting from the addition of sequences for respective restriction enzymes (3xFLAG: MDYKDHDGDYKDHDIDYKDDDDKL, 6His-c-myc: MAHHHHHHASEQKLISEEDLIKDPN, 6His-HA: MAHHHHHHASYPYDVPDYAPEYTDPN). All plasmids constructed in this study are listed in Table 2. 
Table 2 Plasmids constructed for generation of stably transfected cell lines

\begin{tabular}{lll}
\hline Plasmid used for transfection & Original vector (source) & Human UGT coding sequences cloned in MCS \\
\hline HsUGTGolgi1 & 3xFLAG-myc-CMV-26 (Sigma) & UGT1 splice variant with FLAG epitope at N-terminus \\
HsUGTGolgi2 & pVitro1-neo (InvivoGen) & MCS1: UGT1 splice variant with 6His-HA epitope at N-terminus \\
HsUGTER & 3xFLAG-myc-CMV-26 (Sigma) & UGT2 splice variant with FLAG epitope at N-terminus \\
HsUGTER/Golgi & pVitro1-neo (InvivoGen) & MCS1: UGT2 splice variant with 6His-HA epitope at N-terminus; MCS2: \\
& & UGT1 splice variant with 6His-c-myc epitope at N-terminus
\end{tabular}

Cell maintenance and transfection

MDCK and MDCK-RCA ${ }^{\mathrm{r}}$ mutant cells were grown in minimum essential medium (MEM, Sigma-Aldrich) supplemented with $10 \%$ fetal bovine serum, $2 \mathrm{mM} \mathrm{L-}$ glutamine, $100 \mathrm{IU}$ penicillin/ml and $100 \mu \mathrm{g} / \mathrm{ml}$ streptomycin (Sigma-Aldrich). $\mathrm{CHO}$ and CHO-Lec8 mutant cells were grown in MEM with alpha-modification ( $\alpha$-MEM, SigmaAldrich), supplemented with $10 \%$ fetal bovine serum, $2 \mathrm{mM}$ L-glutamine, $100 \mathrm{IU}$ penicillin/ml and $100 \mu \mathrm{g} / \mathrm{ml}$ streptomycin. Alternatively, wild-type MDCK and MDCK-RCA ${ }^{\mathrm{r}}$ mutant cells were grown in EX-CELL MDCK serumfree medium (Sigma-Aldrich) supplemented with $6 \mathrm{mM}$ L-glutamine, $100 \mathrm{IU}$ penicillin $/ \mathrm{ml}$ and $100 \mu \mathrm{g} / \mathrm{ml}$ streptomycin. $\mathrm{CHO}$ and CHO-Lec8 mutant cells were grown in SFM4CHO-A serum-free medium (Thermo Scientific) supplemented with $4 \mathrm{mM}$ L-glutamine, $100 \mathrm{IU}$ penicillin $/ \mathrm{ml}$ and $100 \mu \mathrm{g} / \mathrm{ml}$ streptomycin. Cells were grown in a humidified incubator $\left(37^{\circ} \mathrm{C}, 5 \% \mathrm{CO}_{2}\right)$.

MDCK-RCA ${ }^{\mathrm{r}}$ and CHO-Lec8 mutant cells were transfected with expression plasmids (Table 2) using ESCORT ${ }^{\mathrm{TM}}$ IV Transfection Reagent (Sigma-Aldrich) according to the manufacturer's instructions. Stable transfectants were selected in complete media containing $200(\mathrm{MDCK}) / 150$ (CHO) $\mu \mathrm{g} / \mathrm{ml}$ zeocin or 600 (MDCK) $/ 500$ (CHO) $\mu \mathrm{g} / \mathrm{ml}$ G-418 sulfate (InvivoGen).

\section{Cell lysis and protein determination}

Cells adapted to serum-free culture conditions were collected and lysed using Complete Lysis-M reagent (Roche) supplemented with protease inhibitor cocktail and 1 mM EDTA according to the manufacturer's instructions. Protein concentration was determined using the modified Bradford method [44].

Detection of epitope-tagged proteins by Western blotting

Aliquots containing $20 \mu \mathrm{g}$ of total proteins were separated by SDS-PAGE in $8 \%$ gels and transferred onto nitrocellulose membranes (Whatman). For detection of the HA epitope, membranes were blotted with a 1:1,000 dilution of mouse horseradish peroxidase (HRP)-conjugated antiHA antibody (Roche). For detection of the FLAG epitope, membranes were blotted with a 1:1,000 dilution of mouse anti-FLAG monoclonal antibody (Sigma-Aldrich), followed by incubation with a 1:10,000 dilution of HRP-conjugated goat anti-mouse antibody (Promega). For detection of the c-myc epitope, membranes were blotted with a $1: 1,000$ dilution of mouse anti-c-myc monoclonal antibody (SigmaAldrich), followed by incubation with a 1:10,000 dilution of HRP-conjugated goat anti-mouse antibody. Immunoreactive bands were visualized using the Western Lightning Chemiluminescence Reagent Plus system (Perkin Elmer).

Analysis of glycoproteins with lectins

Aliquots containing $20 \mu \mathrm{g}$ of total proteins extracted from cells grown in respective serum-free media were separated by SDS-PAGE in $8 \%$ gels and transferred onto nitrocellulose membranes. After blocking with Carbo-Free Blocking Solution (Vector Laboratories) membranes were incubated with appropriate biotinylated lectins (Vector Laboratories) in $50 \mathrm{mM}$ Tris- $\mathrm{HCl}, \mathrm{pH} 7.5$, containing $150 \mathrm{mM} \mathrm{NaCl}$ (TBS), $0.2 \%$ Tween-20, $1 \mathrm{mM} \mathrm{Mg}^{2+}$, $1 \mathrm{mM} \mathrm{Ca}^{2+}$ and $1 \mathrm{mM} \mathrm{Mn}^{2+}$. Lectins bound to specific glycans were subsequently detected using alkaline phosphatase-conjugated avidin D (Vector Laboratories) and visualized with NBT/BCIP solution (Roche) according to the manufacturer's instructions.

Isolation of $\mathrm{N}$-glycans from MDCK and $\mathrm{CHO}$ cells

Cells $\left(\sim 10^{7}\right)$ grown in respective serum-free media were scraped from tissue culture plates, washed twice with $15 \mathrm{ml}$ of cold PBS and collected at $200 \times g$ for $5 \mathrm{~min}$. Then, cells were lysed on ice in $500 \mu \mathrm{l}$ of lysis buffer $(20 \mathrm{mM}$ Tris$\mathrm{HCl} \mathrm{pH} 7.4,150 \mathrm{mM} \mathrm{NaCl}$ ), containing 1\% Nonidet NP-40 and Complete protease inhibitors (without EDTA) for $1 \mathrm{~h}$. After incubation in lysis buffer, samples were additionally sonicated 3 times, each time with $30 \mathrm{~s}$ pulses and 2-min cooling between pulses. Lysates were centrifuged at $18,000 \times g$ for $15 \mathrm{~min}$ at $4^{\circ} \mathrm{C}$. Cleared supernatants were used to isolate the total pool of glycoproteins. For this 
purpose, glycoproteins were bound using spin columns (Sigma) containing $300 \mu \mathrm{l}$ of mixed beads $(1: 1, v / v)$ of WGA-agarose and ConA-agarose (Vector Laboratories). Packed columns were washed 4 times with $500 \mu \mathrm{l}$ of equilibration buffer $(20 \mathrm{mM}$ Tris- $\mathrm{HCl} \mathrm{pH} 7.4$, containing $1 \mathrm{M} \mathrm{NaCl}, 1 \mathrm{mM} \mathrm{MnCl} 2,1 \mathrm{mM} \mathrm{CaCl}, 1 \mathrm{mM} \mathrm{MgCl} 2$ ), each time for $1 \mathrm{~min}$ at $1,000 \times \mathrm{g}$. Cell lysates were loaded onto columns and incubated for $30 \mathrm{~min}$ at $4^{\circ} \mathrm{C}$ with slow rotation. After binding, columns were centrifuged at $1,000 \times g$ for $1 \mathrm{~min}$ and supernatants were discarded. Lectin beads were washed 3 times with equilibration buffer, each time for 10 min with slow rotation. Finally, glycoproteins were released sequentially from lectin affinity gel with $2 \times$ $250 \mu \mathrm{l}$ of equilibration buffer containing $500 \mathrm{mM} \alpha$ mannopyranoside (Sigma) and $2 \times 250 \mu \mathrm{L}$ of the same buffer containing $800 \mathrm{mM} N$-acetylglucosamine (Sigma), with 15-min incubation in each elution step. Glycoprotein samples were concentrated to $50 \mu \mathrm{l}$ by ultrafiltration using Microcon 10 (Millipore). During this procedure, elution buffer was changed to glycoprotein denaturation buffer $(\mathrm{N}$-glycosidase $\mathrm{F}$ deglycosylation enzyme pack, New England Biolabs). Acetone precipitation of cellular proteins was also performed as an alternative method to obtain concentrated samples for subsequent deglycosylation. Whole-cell lysate proteins were diluted to $2 \mathrm{mg} / \mathrm{ml}$ using the lysis buffer and $500 \mu \mathrm{l}$ aliquots were precipitated overnight at $-20^{\circ} \mathrm{C}$ with an equal volume of cold acetone. After centrifugation at $10,000 \times g$ for $10 \mathrm{~min}$, precipitates were air-dried and resuspended in glycoprotein denaturation buffer ( $N$-glycosidase $\mathrm{F}$ deglycosylation enzyme pack). Deglycosylation was performed according to the manufacturer's protocol using $1 \mu \mathrm{l}$ of the enzyme (500 U) and incubation for $18 \mathrm{~h}$ at $37^{\circ} \mathrm{C}$ in deglycosylation buffer. Both methods of glycoproteins isolation resulted in a similar glycan pool. Released $N$-glycans were isolated on $50 \mathrm{mg}$ Hypercarb graphite SPE columns (Thermo Electron) using the protocol described for GlycoClean H SPE columns I (Glyko) and dried using a Maxi Dry Lyo (Heto). $N$-glycans were fluorescently labeled with 2-aminobenzamide (2-AB) according to the reported protocol [45] and purified on a 1-mm 3 MM blotting filter paper (Whatman) placed on the bottom of an empty glass column ( $1 \mathrm{~cm}$ diameter) with the procedure identical to the protocol described for GlycoClean S cartridges (Glyko). Small fractions of all samples ( $\sim 5-15 \%)$ were separated on a GlycoSep N normal-phase column (Glyko) using the high salt solvent system (HSSS) as previously described [46] and the Perkin Elmer Series 200 HPLC system (Perkin Elmer) equipped with a 200a fluorescence detector (Perkin Elmer). Exoglycosidase digestion was performed as previously described [46]. Reactions were performed sequentially in $50 \mu$ lof the universal buffer (0.1 M citrate-phosphate buffer, $\mathrm{pH}$ 5.5). After first digestion with unspecific neuraminidase (New
England Biolabs), samples were filtered using Ultrafree MC spin columns (Millipore). Membranes were washed twice with $100 \mu \mathrm{l}$ of Milli-Q water. Samples were dried and resuspended in $100 \mu \mathrm{l}$ of Milli-Q water. Half of the sample volume $(50 \mu \mathrm{l})$ was dried, dissolved in $10 \mu \mathrm{l}$ of $70 \%$ acetonitrile, and separated on a GlycoSep N column using high-performance liquid chromatography (HPLC). The rest of the sample was digested with 1-3,4 $\beta$-galactosidase (New England Biolabs), filtered using an Ultrafree MC spin column, dried, re-suspended in $10 \mu \mathrm{l}$ of $70 \%$ acetonitrile, .and separated on a GlycoSep N column using HPLC.

\section{Analysis of proteoglycans by Western blotting}

Aliquots containing $20 \mu \mathrm{g}$ of total proteins extracted from cells grown in respective serum-free media were separated by SDS-PAGE in $8 \%$ gels and transferred onto nitrocellulose membranes (Whatman). For the detection of keratan sulfate, membranes were blotted with a 1:10,000 dilution of mouse anti-keratan sulfate monoclonal antibody (Chemicon), followed by incubation with a 1:10,000 dilution of HRPconjugated goat anti-mouse antibody. For the detection of chondroitin-4-sulfate, samples were subjected to prior digestion with $0.2 \mathrm{U} / \mathrm{ml}$ of chondroitinase $\mathrm{ABC}$ from Proteus vulgaris (Sigma-Aldrich) in $50 \mathrm{mM}$ Tris- $\mathrm{HCl}$, containing $60 \mathrm{mM}$ sodium acetate for $24 \mathrm{~h}$ at $37^{\circ} \mathrm{C}$. After digestion, which exposed the epitopes, aliquots containing $15 \mu \mathrm{g}$ of proteins were separated by SDS-PAGE in $8 \%$ gels and transferred onto nitrocellulose membranes. Membranes were blotted with a 1:10,000 dilution of mouse anti-chondroitin-4-sulfate monoclonal antibody (Chemicon), followed by incubation with a 1:10,000 dilution of HRP-conjugated goat anti-mouse antibody. Immunoreactive bands were visualized using the Western Lightning Chemiluminescence Reagent Plus system.

\section{Results and discussion}

\section{Identification of the second UGT isoform in MDCK} and $\mathrm{CHO}$ cell lines

Previously, two UGT isoforms (UGT1 and UGT2) have been found in humans [22, 24], whereas only UGT2-like isoform in the CHO cell line [25] and UGT1-like isoform in the MDCK cell line [34] and in mouse [35] have been identified. In this study, using a modified RACE technique, we identified for the first time sequences of UGT1 and UGT2-like splice variants in CHO and MDCK cells, respectively [EMBL: FN825776, EMBL:FN825775]. It is likely that former difficulties in the detection of the second splice variant of UGT in cells other than human cells could result from lower levels of the respective transcripts. 
Analysis of their mRNA showed that independently of a mammalian cell, although ORFs are similar in length, the transcript encoding the UGT2 version is significantly shorter $(\sim 1,500 \mathrm{bp})$ compared to the UGT1 version $(\sim 2,300 \mathrm{bp})$, the latter containing a longer non-coding $3^{\prime}$ region $(\sim 1,200$ bp for UGT1 compared to $\sim 300$ bp for UGT2). It seems that this may be a general feature of UGT isoform coding sequences. In accordance with the published data $[25,26]$ also the UGT2-like form found in MDCK cells contained a dilysine motif at the $\mathrm{C}$ terminus (LLTKVKGS).

Tissue distribution and expression of mammalian UGT isoforms

Oelmann et al. [25] suggested that differences in UGT isoform expression may reflect tissue- and/or speciesspecific regulation processes. However, no studies have been performed to support this hypothesis. To compare tissue distribution of both UGT isoforms, both transcripts were analyzed in 20 human tissues and 9 human cell lines using two-step RT-PCR. To avoid potential amplification of PCR products from DNA template, all RNA samples were digested with DNase. In control PCR reactions carried out with purified RNA as a template, no products were detected. Primers were designed to give specific products of the desired length from the cDNA template only. As shown in Fig. 1a and b, abundant relative expression of the UGT2 version, compared to the UGT1 version, was observed in almost all tissues and cell lines examined. It is likely that, compared to UGT2, the UGT1 variant may be dominant in tissues characterized by intensive metabolism (e.g. kidney, liver, or placenta) and/or tissues composed of high levels of proteoglycans such as keratan sulfate (e.g. trachea). Relative levels of mRNA encoding UGT1 and UGT2 isoforms found in CHO cells were comparable and similar to those found in several human tissues and cell lines (Fig. 1c). In contrast, in MDCK cells a lower level of mRNA encoding the UGT2 splice variant was observed compared to the UGT1 transcript level (Fig. 1c). This suggests that this cell line may exhibit distinct metabolism of galactosylated macromolecules.

Analysis of glycoprotein glycosylation profiles of MDCK and CHO wild-type and mutant cells

MDCK-RCA ${ }^{\mathrm{r}}$ and CHO-Lec8 mutant cells are significantly defective in the transport of UDP-galactose [22, 29, 36]. It has been shown by lectin staining that both cell lines exhibit a $70-90 \%$ deficiency of galactose attached to glycoproteins, glycolipids, and some proteoglycans [28-31, 36]. Glycoproteins produced in CHO-Lec8 and MDCK-RCA ${ }^{\mathrm{r}}$ mutant cells

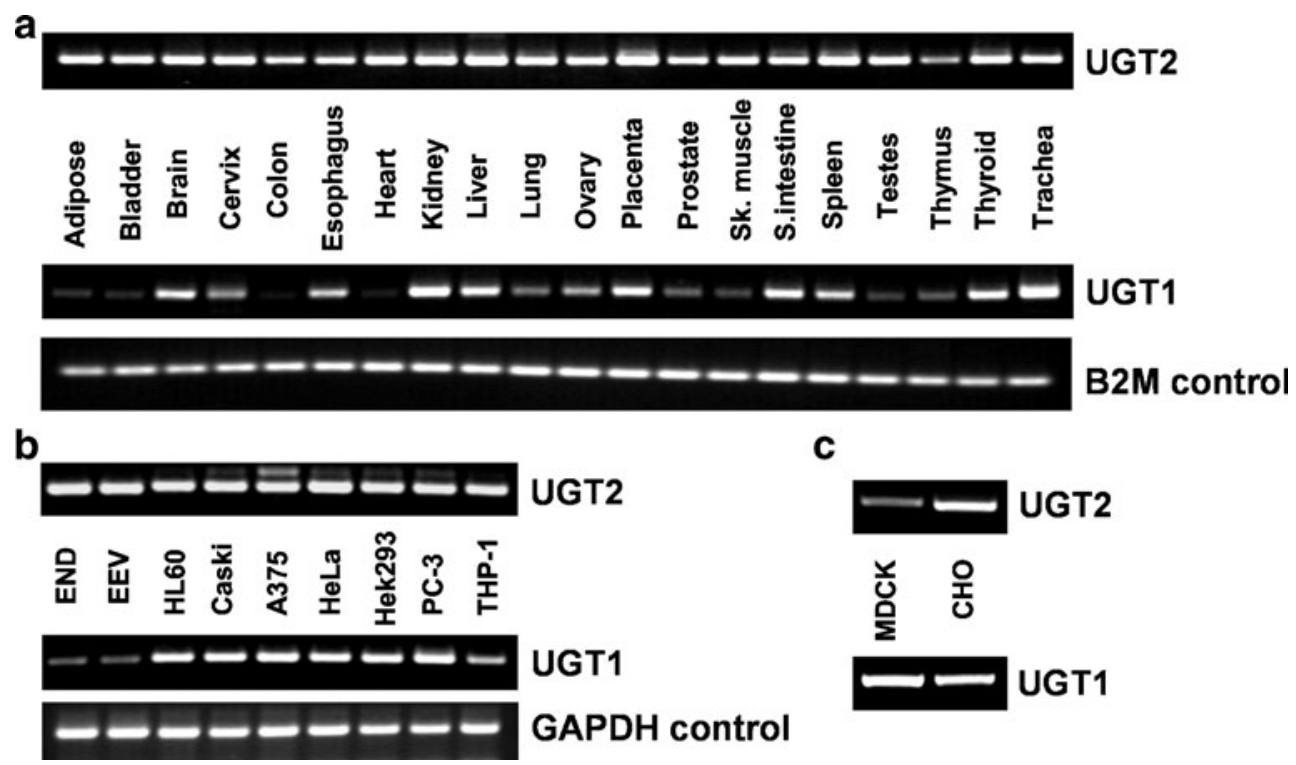

Fig. 1 Tissue distribution of mRNA encoding mammalian UGT splice variants. mRNA levels were analyzed in selected human tissues (a), selected human cell lines (b), and MDCK and CHO cell lines (c) using RT-PCR. Resulting products were visualized in agarose gels with ethidium bromide. RNA integrity was examined using an Agilent Technologies Bioanalyzer (see Supplementary Fig. S1). Expression levels of housekeeping genes, beta-2-microglobulin (B2M) or glyceraldehyde-3-phosphate dehydrogenase (GAPDH) are shown.
Similar patterns were obtained in all reactions repeated at least two times. UGT1 and UGT2, UDP-galactose transporter splice variants; END and EEV, cell lines derived from normal human endometrium; HL60, human promyelocytic leukemia cells; CaSki, human cervical carcinoma cell line; A375, human melanoma cell line; HeLa, human cervical carcinoma cell line; Hek293, human embryonic kidney cell line; PC-3, human prostate cancer cell line; THP-1, human acute monocytic leukemia cell line 

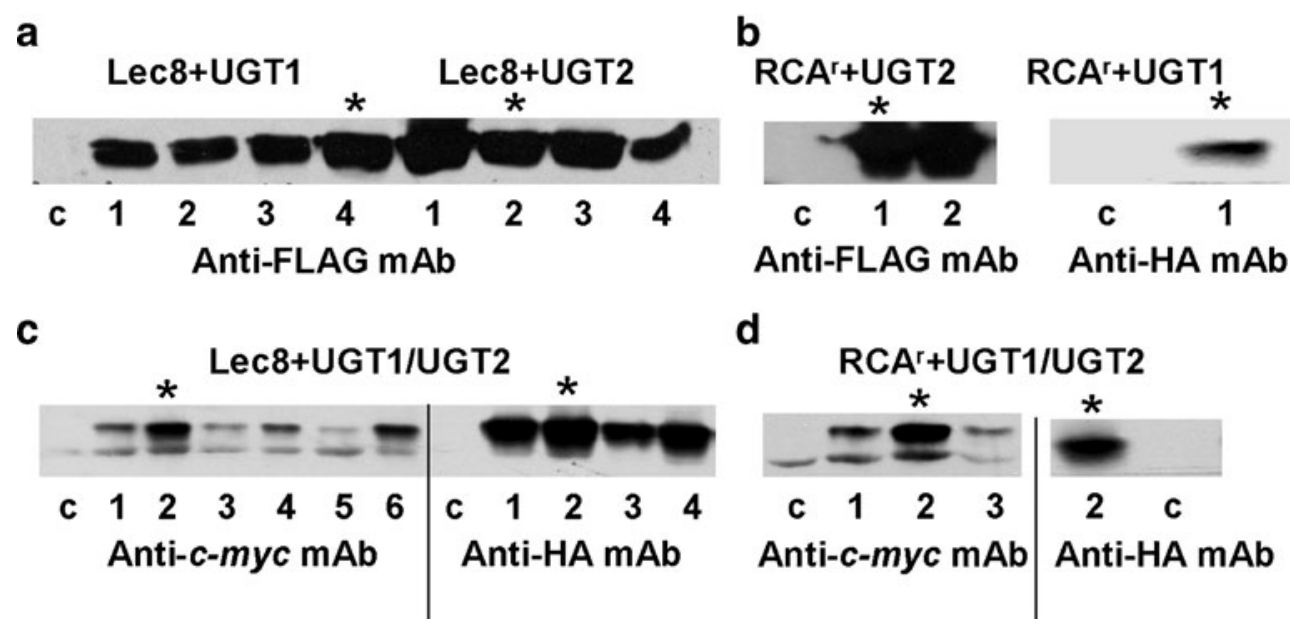

Fig. 2 Analysis of UGT splice variants expression in CHO-Lec8 and MDCK-RCA ${ }^{\mathrm{r}}$ mutant cell lines. Mutant cells were stably transfected with respective expression plasmids and UGT splice variants expressed either singly (a and b) or in combination (c and d) were identified using Western blotting with antibodies against epitopes as follows: for single expression in CHO-Lec8 cells UGT1 and UGT2 contained the FLAG epitope; for single expression in MDCK-RCA ${ }^{\mathrm{r}}$ cells UGT1 contained the 6His-HA epitope and UGT2 contained the
FLAG epitope; for dual expression in CHO-Lec8 cells UGT1 contained the 6His-c-myc epitope and UGT2 contained the 6His-HA epitope; and for dual expression in MDCK-RCAr cells UGT1 contained the 6 His-c-myc epitope and UGT2 contained the 6His-HA epitope. Numbers indicate positive clones expressing the respective proteins obtained in representative experiments. Data presented in this study were obtained from experiments using clones indicated with an asterisk. C, control cells transfected with empty vector

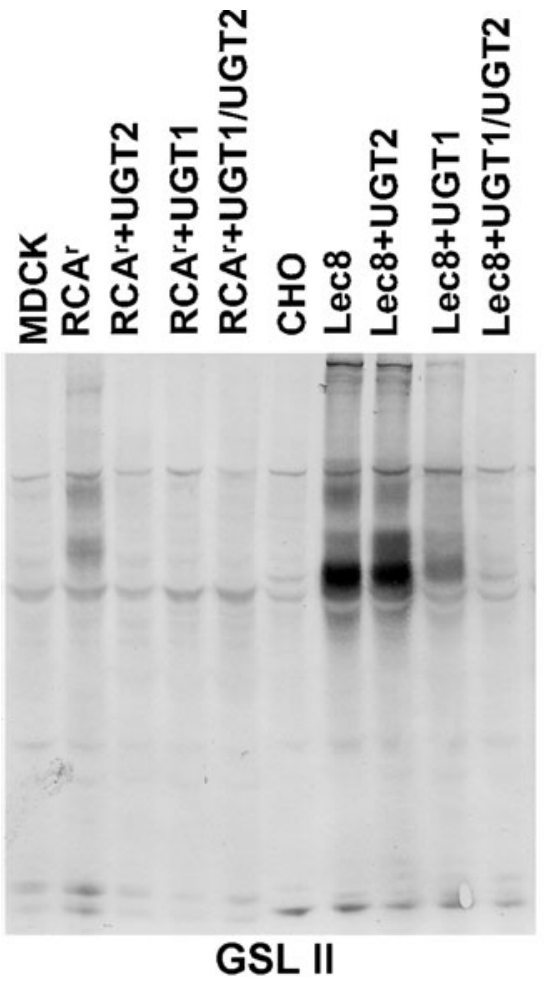

Fig. 3 Lectin staining of glycoproteins derived from wild-type MDCK and CHO and mutant MDCK-RCA ${ }^{\mathrm{r}}$ and CHO-Lec8 cells. Mutant cells were stably transfected with respective expression plasmids and UGT splice variants were expressed either singly or in combination. Proteins were extracted as described in Materials and Methods, separated by SDS-PAGE and transferred onto nitrocellulose membranes. Phenotypic correction was determined using GSL II (lectin from Griffonia simplicifolia) or VVL (lectin from Vicia villosa)
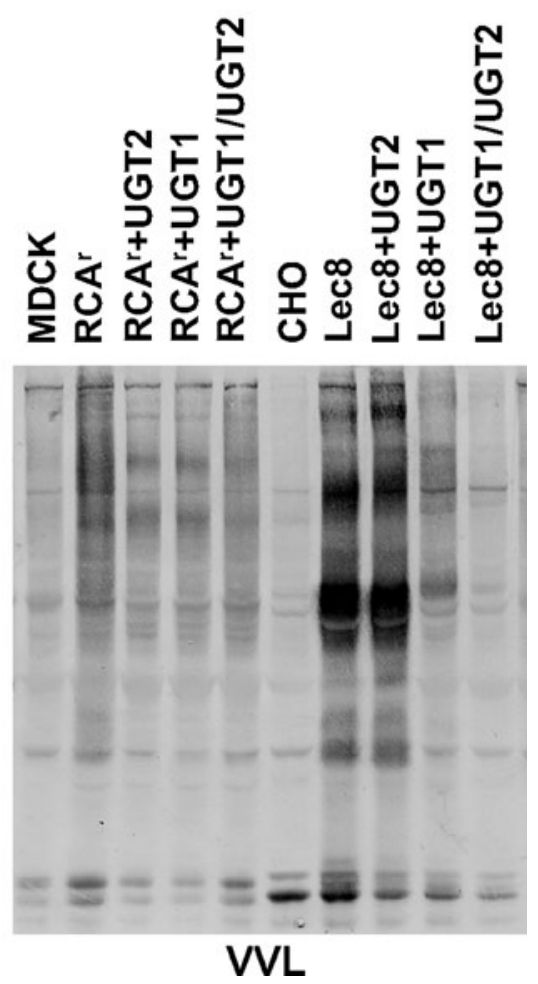

lectin. Reactivity of glycoproteins derived from protein cell extracts with GSL II, lectin specific for terminal $N$-acetylglucosamine present in both $N$ - and $O$-glycans or with VVL, lectin specific for $N$ acetylgalactosamine attached to $O$-glycans was visualized using alkaline phosphatase-conjugated avidin D and NBT/BCIP. Control cells transfected with empty vector gave similar results as mutant cells. Representative data out of three sets with a similar pattern are shown 
are also deficient in sialic acid, since this sugar is most often attached to galactose residues. Detailed glycosylation analysis has been performed in murine Had-1 mutant cells and their parental FM3A cells [35], as well as in CHO-Lec8 mutant cells [37, 38].

To date, the role of both splice variants of UGT in galactosylation of macromolecules in $\mathrm{CHO}$ and MDCK cells has not been reported. Therefore, in this study we developed an experimental model of UGT functional analysis using overexpression of both human splice variants, singly or in combination, in the MDCK-RCA ${ }^{\mathrm{r}}$ and CHO-Lec8 mutant cells. Several clones expressing native and $\mathrm{N}$-tagged proteins, varying in expression levels of respective UGT splice variants (Fig. 2 and data not shown), were further examined in regard to phenotypic correction. Results of phenotypic correction analyzed by lectin staining were similar for each clone examined; therefore in this study data for selected tagged UGTs (indicated in Fig. 2 with asterisks), which can be easily monitored with antibodies, were shown. All overexpressed proteins examined in this study properly localized to the ER and/or Golgi apparatus and transported UDP-Gal into Golgi vesicles (unpublished data). To date, all analyses of glycoprotein glycosylation have been performed using cells cultured in the presence of serum [e.g. 32, 35, 37, 38]. In our preliminary studies we found that serum glycoconjugates attached to the cell surface were unable to be completely removed by washing, which resulted in partial masking of endogenous glycosylation profiles of the cells. Therefore, to analyze glycosylation profiles of glycoproteins derived from the cells only, all cells were cultured in serum-free medium. When the cells were stably transfected with UGT coding sequences, the mutant cells exhibited a phenotype similar to wild-type cells, as analyzed by reactivity with specific lectins. As shown in
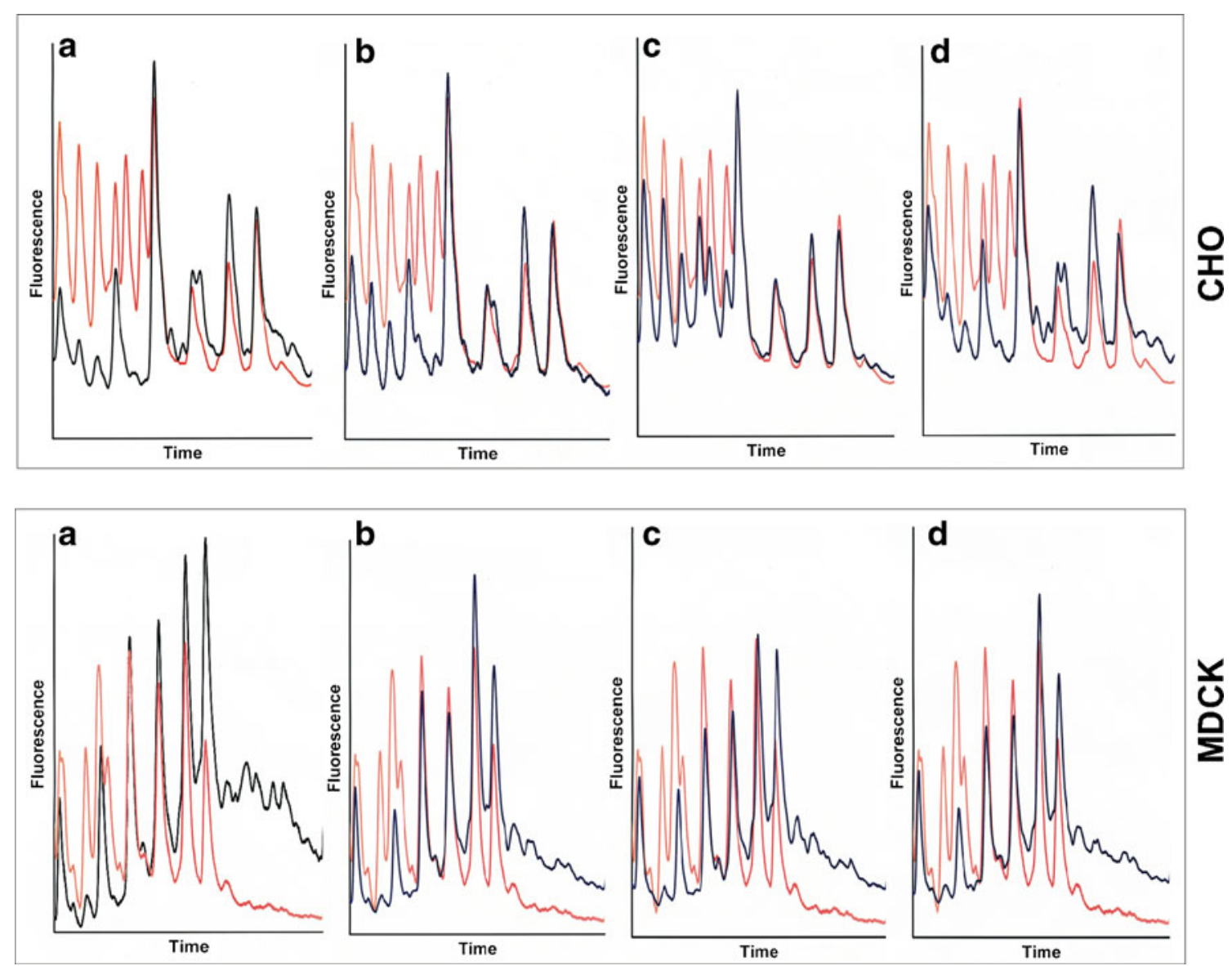

Fig. $4 \mathrm{~N}$-glycan profiles of wild-type MDCK and $\mathrm{CHO}$ and mutant MDCK-RCA ${ }^{\mathrm{r}}$ and CHO-Lec8 cells. $N$-glycans were released from glycoproteins using $N$-glycosidase F, fluorescently labeled with 2-AB, and separated using the GlycoSep $\mathrm{N}$ column. a $N$-glycan profiles of wild-type $\mathrm{CHO}$ or MDCK cells (black lines) and mutant CHO-Lec8 or MDCK-RCA ${ }^{\mathrm{r}}$ cells (red lines). b $\mathrm{N}$-glycan profiles of CHO-Lec8 or MDCK-RCA ${ }^{\mathrm{r}}$ mutant cells (red lines) and the mutant cells expressing UGT1 isoform (black lines). c $\mathrm{N}$-glycan profiles of CHO-Lec8 or

MDCK-RCA ${ }^{\mathrm{r}}$ mutant cells (red lines) and the mutant cells expressing UGT2 isoform (black lines). d $N$-glycan profiles of CHO-Lec8 or MDCK-RCA ${ }^{r}$ mutant cells (red lines) and the mutant cells expressing both UGT1 and UGT2 isoforms (black lines). Control cells transfected with empty vector gave similar results as mutant cells. Representative data out of three sets with a similar pattern are shown. Fluorescence, fluorescence detector response; Time, time [min] 
Fig. 3, analysis carried out with GSL II, a lectin specific for terminal $N$-acetylglucosamine (GlcNAc) present in both $N$ and $O$-glycans, resulted in more intense signals in mutant cells, especially in CHO-Lec8 cells. This effect results from increased levels of truncated oligosaccharide chains terminated with GlcNAc in the mutant cells possessing lower levels of galactose attached to oligosaccharide chains. In the case of MDCK-RCA ${ }^{\mathrm{r}}$ mutant cells, overexpression of the UGT1 and/or UGT2 splice variant resulted in correction of the $\mathrm{N}$-glycosylation defect (Fig. 3). In contrast, CHO-Lec8 mutant cells regained a glycosylation pattern more similar to wild-type cells after correction with both splice variants only. This suggests that $\mathrm{CHO}$ cells require both UGT splice variants to perform proper galactosylation of glycoproteins. One may conclude that more efficient correction in MDCK-RCA ${ }^{\mathrm{r}}$ mutant cells may result from the presence of the additional, shorter version of UGT [34]. We previously found that mRNA levels of this UGT variant were higher in the MDCK$\mathrm{RCA}^{\mathrm{r}}$ mutant cells, which may additionally support the involvement of this UGT variant in UDP-galactose transport.

Analysis with VVL, a lectin specific for $\mathrm{N}$-acetylgalactosamine (GalNAc) attached to $O$-glycans, is shown in Fig. 3. This sugar is more exposed in cells lacking proper attachment of galactose to some types of $O$-glycans. The effect of correction of the mutation effect in both mutant cell lines was similar to that analyzed with GSL II, although the intensity of the reaction was higher.

The second approach consisted of comparison of $\mathrm{N}$ glycosylation profiles of fluorescently labeled glycans released from glycoproteins and separated on a GlycoSep N column, which was calibrated with partially digested 2-AB dextran. The normal-phase HPLC separates 2-AB-labeled $\mathrm{N}$-glycans based on differences in chain length, as shorter oligosaccharides elute earlier than full-length structures. Data shown in Fig. 4a (top panel) demonstrate differences in glycosylation profiles observed between the wild-type $\mathrm{CHO}$ and mutant CHO-Lec8 cells. Although the differences between wild-type MDCK and mutant MDCK-RCA ${ }^{\mathrm{r}}$ cells are less significant, they are easily detectable (Fig. 4a, bottom panel). One may conclude that this difference could result from the presence of the additional, shorter splice variant of UGT found only in MDCK cells, including the mutant MDCK-RCA ${ }^{\mathrm{r}}$ cells, which would participate in galactosylation of macromolecules. Compared with the wild-type cells, in both examined mutant cells truncated oligosaccharides comprise the major pool of $\mathrm{N}$-glycans. This effect is similar to that demonstrated in Had-1 cells [35]. Analysis of glycosylation profiles after phenotypic correction of the mutant cells further demonstrated that both UGT1 and UGT2 splice variants were able, at least in part, to correct the galactosylation defect in both mutant cell lines (Fig. 4b-d). This was visible in the increased pool of more complex oligosaccharides.
To detect whether UGT is the only transporter responsible for galactose delivery for subsequent galactosylation in MDCK and CHO cells, we compared $N$-glycosylation profiles of the mutant cells before and after galactosidase treatment. Since sialic acid residues attached to the terminal galactose may block galactosidase access, prior neuraminidase treatment was performed. After galactosidase treatment a slight decrease in the range comprising higher-molecularweight glycans and a slight increase in the range comprising lower-molecular-weight, truncated glycans were observed in both mutant cells (data not shown). The effect was not related to the specific type of glycans, suggesting that in the mutant cells low galactose levels are still present in $N$-glycans attached to glycoproteins. Previously, Hara et al. [35] demonstrated that also in murine Had-1 mutant cells lacking functional UGT some galactosylation occurs. All these data indicate that an additional mechanism of UDP-Gal transport from the cytosol to the Golgi apparatus may exist. Although an alternative mechanism of UDP-galactose transport in CHO-Lec8 mutant cells is not known, in MDCK-RCA ${ }^{\mathrm{r}}$ mutant cells the additional, shorter UGT form [34] may support UDP-galactose transport, at least in part, leading to partial galactosylation of glycoproteins.
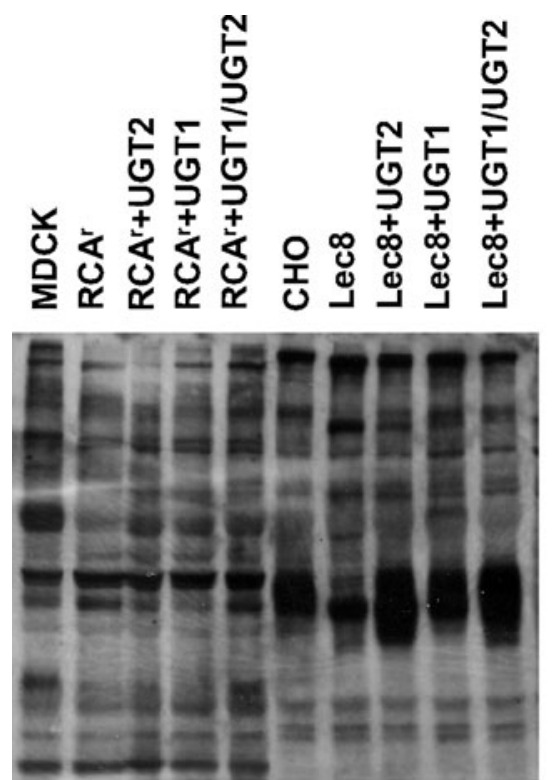

Chondroitin-4-sulfate

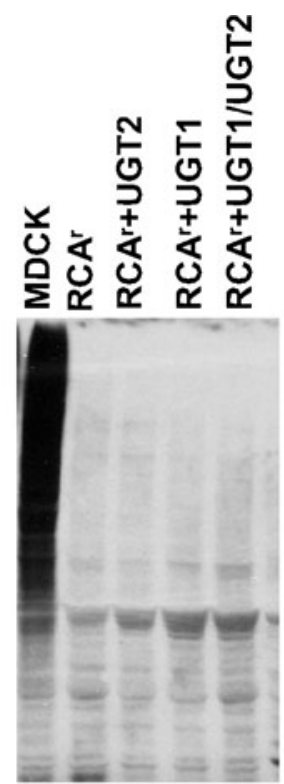

keratan sulfate

Fig. 5 Analysis of proteoglycans produced in wild-type MDCK and $\mathrm{CHO}$ and mutant MDCK-RCA ${ }^{\mathrm{r}}$ and CHO-Lec8 cells. Mutant cells were stably transfected with respective expression plasmids and UGT splice variants were expressed either singly or in combination. Proteoglycans present in protein cell extracts were separated by SDS-PAGE in their native form (keratan sulfate) or after limited digestion with chondroitinase (chondroitin-4-sulfate) and detected with specific antibodies using Western blotting. Control cells transfected with empty vector gave similar results as mutant cells. Representative data out of three sets with similar pattern are shown 
Analysis of proteoglycans in MDCK and CHO wild-type and mutant cells

Proteoglycans, which play a crucial role in chondrogenesis and skeletal development, belong to a family of macromolecules composed of glycosaminoglycan chains of a repeated disaccharide linked to a central core protein. Within proteoglycans, keratan sulfate contains galactose in long chains, whereas in other types of proteoglycans (chondroitin and heparan sulfates) galactose moieties are attached to the core part of the polysaccharide chain. To examine involvement of the respective UGT splice variants in proteoglycan synthesis, selected proteoglycans were analyzed in MDCK-RCA ${ }^{\mathrm{r}}$ and CHO-Lec8 mutant cells subjected to phenotypic correction. It is worth noting that in both mutant cell lines reactivity with antibodies against chondroitin-4-sulfate was relatively high and only slightly lower compared to the wild-type cells (Fig. 5). A similar effect was demonstrated in radiolabeled MDCK cells [29]. Analysis of chondroitin-4-sulfate demonstrated that correction of the mutant phenotype of CHO-Lec8 and MDCK-RCA ${ }^{\mathrm{r}}$ cells was observed after parallel overexpression of both UGT splice variants, with the most pronounced effect observed in CHO-Lec8 mutant cells (Fig. 5). These data suggest that in synthesis of proteoglycans an additional UDP-galactose transport mechanism may also exist.

CHO cells do not possess keratan sulfate [3,29, and our data not shown]; therefore we analyzed proteoglycans using specific antibodies in MDCK cells only. As shown in Fig. 5, MDCK-RCA ${ }^{\mathrm{r}}$ mutant cells do not produce keratan sulfate, and surprisingly, this effect was not corrected by UGT splice variants overexpressed either singly or in combination. It is likely that MDCK-RCA ${ }^{\mathrm{r}}$ mutant cells may possess an additional, not yet identified, defect within the keratan sulfate biosynthesis pathway. To exclude additional mutations in MDCK-RCA ${ }^{\mathrm{r}}$ cells, we examined in both MDCK and MDCK-RCA ${ }^{\mathrm{r}}$ cells known coding sequences or identified coding sequences for several proteins involved in proteoglycan biosynthesis. However, we were not able to identify a defect in the coding sequences of analyzed mRNA. Analysis of protein similar to sulfotransferase 1 (cloned, sequenced, and submitted to database; EMBL ID: FR775794), protein similar to sulfotransferase 6 (cloned, sequenced, and submitted to database; EMBL ID: FR775795), protein similar to beta-1,4-galactosyltransferase 4 (cloned, sequenced, and submitted to database; EMBL ID: AM989461), UDP-GlcNAc transporter (EMBL ID: AF057365), UDP-GlcNAc:betaGal beta-1,3- $N$-acetylglucosaminyltransferase 2 (NCBI ID: XP_531841), and PAPS transporter 1 (EMBL ID: EF568109) showed that all amino acid sequences were identical in the wild-type and mutant cells.

\section{Conclusions}

Both MDCK-RCA ${ }^{\mathrm{r}}$ and CHO-Lec8 mutant cell lines serve as widely used models to study UDP-galactose transport in mammals. In this study we have identified the second UGT splice variant in CHO (UGT1) and MDCK (UGT2) cells. It is highly possible that UGT1 and UGT2 splice variants are expressed in most (if not all) mammalian cells. We demonstrated that both UGT splice variants are important for glycosylation of proteins. In contrast to MDCK cells, MDCK-RCA ${ }^{\mathrm{r}}$ mutant cells do not produce keratan sulfate and this effect was not corrected by UGT splice variants overexpressed either singly or in combination. It is likely that MDCK-RCA ${ }^{\mathrm{r}}$ mutant cells may possess an additional, not yet identified, defect within the keratan sulfate biosynthesis pathway.

Acknowledgements This work was supported in part by grants no. N301 012 32/0869 and N N302 101436 from the Ministry of Science and Higher Education, Poland (to MO) and the Hungarian Scientific Research Fund no. 77826 (to LW).

Open Access This article is distributed under the terms of the Creative Commons Attribution Noncommercial License which permits any noncommercial use, distribution, and reproduction in any medium, provided the original author(s) and source are credited.

\section{References}

1. Coates, S., Gurney, T., Sommers, L.W., Yeh, M., Hirschberg, C.B.: Subcellular localization of sugar nucleotide synthetases. J. Biol. Chem. 255, 9225-9229 (1980)

2. Munster, A.K., Eckhardt, M., Potvin, B., Muhlenhoff, M., Stanley, P., Gerady-Schahn, R.: Mammalian cytidine 5'-monophosphate $N$-acetylneuraminic acid synthetase: a nuclear protein with evolutionarily conserved structural motifs. Proc. Natl. Acad. Sci. USA 95, 9140-9145 (1998)

3. Hirschberg, C.B., Robbins, P., Abeijon, C.: Transporters of nucleotide sugars, ATP, and nucleotide sulfate in the endoplasmic reticulum and Golgi apparatus. Annu. Rev. Biochem. 67, 49-69 (1998)

4. Gerardy-Schahn, R., Oelmann, S., Bakker, H.: Nucleotide sugar transporters: biological and functional aspects. Biochimie 83, 775-782 (2001)

5. Caffaro, C.E., Hirschberg, C.B.: Nucleotide sugar transporters of the Golgi apparatus: from basic science to diseases. Acc. Chem. Res. 39, 805-812 (2006)

6. Eckhardt, M., Gotza, B., Gerardy-Schahn, R.: Membrane topology of the mammalian CMP-sialic acid transporter. J. Biol. Chem. 274, 8779-8787 (1999)

7. Abe, M., Hashimoto, H., Yoda, K.: Molecular characterization of Vig4/Vrg4 GDP-mannose transporter of the yeast Saccharomyces cerevisiae. FEBS Lett. 458, 309-312 (1999)

8. Puglielli, L., Hirschberg, C.B.: Reconstitution, identification, and purification of the rat liver Golgi membrane GDP-fucose transporter. J. Biol. Chem. 274, 35596-35600 (1999)

9. Puglielli, L., Mandon, E., Rancour, D.M., Menon, A.K., Hirschberg, C.B.: Identification and purification of the rat liver Golgi membrane 
UDP- $N$-acetylgalactosamine transporter. J. Biol. Chem. 274, 4474 4479 (1999)

10. Gao, X.D., Dean, N.: Distinct protein domains of the yeast Golgi GDP-mannose transporter mediate oligomer assembly and export from the endoplasmic reticulum. J. Biol. Chem. 275, 1771817727 (2000)

11. Hong, K., Ma, D., Beverley, S.M., Turco, S.J.: The Leishmania GDP-mannose transporter is an autonomous, multi-specific, hexameric complex of LPG2 subunits. Biochemistry 39, 2013$2022(2000)$

12. Segawa, H., Kawakita, M., Ishida, N.: Human and Drosophila UDP-galactose transporters transport UDP- $N$-acetylgalactosamine in addition to UDP-galactose. Eur. J. Biochem. 269, 128-138 (2002)

13. Selva, E.M., Hong, K., Baeg, G.H., Beverley, S.M., Turco, S.J., Perrimon, N., Hacker, U.: Dual role of the fringe connection gene in both heparan sulphate and fringe-dependent signalling events. Nat. Cell Biol. 3, 809-815 (2001)

14. Goto, S., Taniguchi, M., Muraoka, M., Toyoda, H., Sado, Y., Kawakita, M., Hayashi, S.: UDP-sugar transporter implicated in glycosylation and processing of Notch. Nat. Cell Biol. 3, 816-822 (2001)

15. Bredeston, L.M., Caffaro, C.E., Samuelson, J., Hirschberg, C.B.: Golgi and endoplasmic reticulum functions take place in different subcellular compartments of Entamoeba histolytica. J. Biol. Chem. 280, 32168-32176 (2005)

16. Berninsone, P.M., Hwang, H.Y., Zemtseva, I., Horvitz, H.R., Hirschberg, C.B.: SQV-7, a protein involved in Caenorhabditis elegans epithelial invagination and early embryogenesis, transports UDP-glucuronic acid, UDP- $N$-acetylgalactosamine, and UDP-galactose. Proc. Natl. Acad. Sci. USA 98, 3738-3743 (2001)

17. Caffaro, C.E., Hirschberg, C.B., Berninsone, P.M.: Independent and simultaneous translocation of two substrates by a nucleotide sugar transporter. Proc. Int. Acad. Sci. USA 103, 16176-16181 (2006)

18. Caffaro, C.E., Luhn, K., Bakker, H., Vestweber, D., Samuelson, J., Berninsone, P.M., Hirschberg, C.B.: A single Caenorhabditis elegans Golgi apparatus-type transporter of UDP-glucose, UDP-galactose, UDP- $N$-acetylglucosamine, and UDP- $N$-acetylgalactosamine. Biochemistry 47, 4337-4344 (2008)

19. Suda, T., Kamiyama, S., Suzuki, M., Kikuchi, N., Nakayama, K., Narimatsu, H., Jigami, Y., Aoki, T., Nishihara, S.: Molecular cloning and characterization of a human multisubstrate specific nucleotide-sugar transporter homologous to Drosophila fringe connection. J. Biol. Chem. 279, 26469-26474 (2004)

20. Ashikov, A., Routier, F., Fuhlrott, J., Helmus, Y., Wild, M., Gerardy-Schahn, R., Bakker, H.: The human solute carrier gene SLC35B4 encodes a bifunctional nucleotide sugar transporter with specificity for UDP-xylose and UDP- $N$-acetylglucosamine. J. Biol. Chem. 280, 27230-27235 (2005)

21. Muraoka, M., Kawakita, M., Ishida, N.: Molecular characterization of human UDP-glucuronic acid/UDP- $N$-acetylgalactosamine transporter, a novel nucleotide sugar transporter with dual substrate specificity. FEBS Lett. 495, 87-93 (2001)

22. Ishida, N., Miura, N., Yoshioka, S., Kawakita, M.: Molecular cloning and characterization of a novel isoform of the human UDP-galactose transporter, and of related complementary DNAs belonging to the nucleotide-sugar transporter gene family. J. Biochem. (Tokyo) 120, 1074-1078 (1996)

23. Kumamoto, K., Goto, Y., Sekikawa, K., Takenoshita, S., Ishida, N., Kawakita, M., Kannagi, R.: Increased expression of UDPgalactose transporter messenger RNA in human colon cancer tissues and its implication in synthesis of Thomsen-Fridenreich antigen and sialyl Lewis a/x determinants. Cancer Res. 61, 4620 4627 (2001)
24. Miura, N., Ishida, N., Hoshino, M., Yamauchi, M., Hara, T., Ayusawa, D., Kawakita, M.: Human UDP-galactose translocator: molecular cloning of a complementary DNA that complements the genetic defect of a mutant cell line deficient in UDP-galactose translocator. J. Biochem. (Tokyo) 120, 236-241 (1996)

25. Oelmann, S., Stanley, P., Gerardy-Schahn, R.: Point mutations identified in Lec8 Chinese hamster ovary glycosylation mutants that inactivate both the UDP-galactose and CMP-sialic acid transporters. J. Biol. Chem. 276, 26291-26300 (2001)

26. Sun-Wada, G.H., Yoshioka, S., Ishida, N., Kawakita, M.: Functional expression of the human UDP-galactose transporters in the yeast Saccharomyces cerevisiae. J. Biochem. (Tokyo) 123, 912-917 (1998)

27. Yoshioka, S., Sun-Wada, G.H., Ishida, N., Kawakita, M.: Expression of the human UDP-galactose transporter in the Golgi membranes of murine Had-1 cells that lack the endogenous transporter. J. Biochem. (Tokyo) 122, 691-695 (1997)

28. Brandli, A.W., Hansson, G.C., Rodriguez-Boulan, E., Simons, K.: A polarized epithelial cell mutant deficient in translocation of UDP-galactose into the Golgi complex. J. Biol. Chem. 263, 16283-16290 (1988)

29. Toma, L., Pinhal, M.A., Dietrich, C.P., Nader, H.B., Hirschberg, C.B.: Transport of UDP-galactose into the Golgi lumen regulates the biosynthesis of proteoglycans. J. Biol. Chem. 271, 3897-3901 (1996)

30. Stanley, P.: Lectin-resistant CHO cells: selection of new mutant phenotypes. Somatic Cell Genet. 9, 593-608 (1983)

31. Stanley, P.: Chinese hamster ovary cell mutants with multiple glycosylation defects for production of glycoproteins with minimal carbohydrate heterogeneity. Mol. Cell. Biol. 9, 377-383 (1989)

32. Briles, E.B., Li, E., Kornfeld, S.: Isolation of wheat germ agglutininresistant clones of Chinese hamster ovary cells deficient in membrane sialic acid and galactose. J. Biol. Chem. 252, 1107-1116 (1977)

33. Ishida, N., Yoshioka, S., Iida, M., Sudo, K., Miura, N., Aoki, K., Kawakita, M.: Indispensability of transmembrane domains of Golgi UDP-galactose transporter as revealed by analysis of genetic defects in UDP-galactose transporter-deficient murine Had-1 mutant cell lines and construction of deletion mutants. J. Biochem. (Tokyo) 126, 1107-1117 (1999)

34. Olczak, M., Guillen, E.: Characterization of a mutation and an alternative splicing of UDP-galactose transporter in MDCK-RCA ${ }^{\mathrm{r}}$ mutant cell line. Biochim. Biophys. Acta 1763, 82-92 (2006)

35. Hara, T., Endo, T., Furukawa, K., Kawakita, M., Kobata, A.: Elucidation of the phenotypic change on the surface of Had-1 cell, a mutant cell line of mouse FM3A carcinoma cells selected by resistance to Newcastle Disease virus infection. J. Biochem. 106, 236-247 (1989)

36. Deutscher, S.L., Hirschberg, C.B.: Mechanism of galactosylation in the Golgi apparatus. A Chinese hamster ovary cell mutant deficient in translocation of UDP-galactose across Golgi vesicle membranes. J. Biol. Chem. 261, 96-100 (1986)

37. Kawar, Z.S., Haslam, S.M., Morris, H.R., Dell, A., Cummings, R.D.: Novel poly-GalNAcbeta1-4GlcNAc (LacdiNAc) and fucosylated poly-LacdiNAc $N$-glycans from mammalian cells expressing beta1,4- $N$-acetylgalactosaminyltransferase and alpha1,3-fucosyltransferase. J. Biol. Chem. 280, 12810-12819 (2005)

38. Stanley, P., Sudo, T., Carver, J.P.: Differential involvement of cell surface sialic acid residues in wheat germ agglutinin binding to parental and wheat germ agglutinin-resistant Chinese hamster ovary cells. J. Cell Biol. 85, 60-69 (1980)

39. Roy, S., Chiba, Y., Takeuchi, M., Jigami, Y.: Characterization of yeast Yea4p, a uridine diphosphate- $N$-acetylglucosamine transporter localized in the endoplasmic reticulum and required for chitin synthesis. J. Biol. Chem. 275, 13580-13587 (2000)

40. Kainuma, M., Chiba, Y., Takeuchi, M., Jigami, Y.: Overexpression of HUT1 gene stimulates in vivo galactosylation by enhancing UDP-galactose transport activity in Saccharomyces cerevisiae. Yeast 18, 533-541 (2001) 
41. Nakanishi, H., Nakayama, K., Yokota, A., Tachikawa, H., Takahashi, N., Jigami, Y.: Hut1 proteins identified in Saccharomyces cerevisiae and Schizosaccharomyces pombe are functional homologues involved in the protein folding process at the endoplasmic reticulum. Yeast 18, 543-554 (2001)

42. Kabuss, R., Ashikov, A., Oelmann, S., Gerardy-Schahn, R., Bakker, H.: Endoplasmic reticulum retention of the large splice variant of UDP-galactose transporter is caused by a dilysine motif. Glycobiology 15, 905-911 (2005)

43. Vandesompele, J., De Preter, K., Pattyn, F., Poppe, B., Van Roy, N., De Paepe, A., Speleman, F.: Accurate normalization of realtime quantitative RT-PCR data by geometric averaging of multiple internal control genes. Genome Biol. 3(7), research0034.10034.11 (2002)

44. Zor, T., Selinger, Z.: Linearization of the Bradford protein assay increases its sensitivity: theoretical and experimental studies. Anal. Biochem. 236, 302-308 (1996)

45. Bigge, J.C., Patel, T.P., Bruce, J.A., Goulding, P.N., Charles, S.M., Parekh, R.B.: Non-selective and efficient fluorescent labeling of glycans using 2-aminobenzamide and anthranilic acid. Anal. Biochem. 230, 229-238 (1995)

46. Olczak, M., Watorek, W.: Structural analysis of $N$-glycans from yellow lupin (Lupinus luteus) seed diphosphonucleotide phosphatase/phosphodiesterase. Biochim. Biophys. Acta 1523, 236-245 (2000) 